\title{
Frontières
}

\section{Les besoins d'apprentissage des bénévoles en contexte de soins palliatifs pédiatriques}

Vers une définition plus précise et une compréhension plus approfondie

\section{Manon Champagne}

Volume 20, numéro 1, automne 2007

La « bonne mort »

URI : https://id.erudit.org/iderudit/017948ar

DOI : https://doi.org/10.7202/017948ar

Aller au sommaire du numéro

Éditeur(s)

Université du Québec à Montréal

ISSN

1180-3479 (imprimé)

1916-0976 (numérique)

Découvrir la revue

Citer cet article

Champagne, M. (2007). Les besoins d'apprentissage des bénévoles en contexte de soins palliatifs pédiatriques : vers une définition plus précise et une compréhension plus approfondie. Frontières, 20(1), 52-61.

https://doi.org/10.7202/017948ar
Résumé de l'article

Le but de la recherche-action dont les résultats sont ici partiellement rapportés consistait à améliorer la formation de bénévoles s'apprêtant à oeuvrer dans un programme de répit à domicile offert à des familles d'enfants atteints d'une maladie à issue fatale. Elle visait notamment à mieux connaître les besoins de formation de ces bénévoles et à cerner les principales composantes d'un programme de formation initiale leur étant destiné. Cet article présente une synthèse des besoins d'apprentissage qui ont été déterminés avec la participation de parents, de bénévoles, de formatrices et d'employées, à l'aide de diverses méthodes de collecte de données. Cette synthèse comprend 27 grands thèmes présentés sous neuf rubriques. En outre, l'auteure discute brièvement ce qui, relativement aux besoins d'apprentissage des bénévoles, caractérise le bénévolat en contexte de soins palliatifs pédiatriques par rapport au bénévolat effectué auprès d’adultes en fin de vie.
Ce document est protégé par la loi sur le droit d'auteur. L'utilisation des services d'Érudit (y compris la reproduction) est assujettie à sa politique d'utilisation que vous pouvez consulter en ligne.

https://apropos.erudit.org/fr/usagers/politique-dutilisation/ 


\section{Résumé}

Le but de la recherche-action dont les résultats sont ici partiellement rapportés consistait à améliorer la formation de bénévoles s'apprêtant à œuvrer dans un programme de répit à domicile offert à des familles d'enfants atteints d'une maladie à issue fatale. Elle visait notamment à mieux connaître les besoins de formation de ces bénévoles et à cerner les principales composantes d'un programme de formation initiale leur étant destiné. Cet article présente une synthèse des besoins d'apprentissage qui ont été déterminés avec la participation de parents, de bénévoles, de formatrices et d'employées, à l'aide de diverses méthodes de collecte de données. Cette synthèse comprend 27 grands thèmes présentés sous neuf rubriques. En outre, l'auteure discute brièvement ce qui, relativement aux besoins d'apprentissage des bénévoles, caractérise le bénévolat en contexte de soins palliatifs pédiatriques par rapport au bénévolat effectué auprès d'adultes en fin de vie.

Mots clés: Soins palliatifs pédiatriques bénévolat - répit - formation -

recherche-action.

\section{Abstract}

The goal of the action research whose results are partially reported here was to improve the training of volunteers wishing to get involved in an In-Home Respite program offered to families of children with degenerative and terminal illnesses. It aimed primarily to provide a better understanding of the training needs of In-Home Respite program volunteers and to identify the main components of an initial training program. This paper presents a synthesis of the learning needs brought forward by parents, volunteers, trainers and employees through the use of different research methods. This synthesis is comprised of 27 themes presented under nine headings. In addition, the author discusses briefly, regarding volunteer learning needs, what characterizes volunteering in a context of pediatric palliative care in comparison to volunteering with adults in end of life situations.

Keywords: Pediatric palliative care volunteering - respite - training action research.

\section{LES BESOINS D'APPRENTISSAGE DES
BÉNÉVOLES EN CONTEXTE
DE SOINS PALLIATIFS
PÉDIATRIQUES} Vers une définition plus précise
et une compréhension plus approfondie

Manon Champagne 1 , Ph. D., professeure associée, École de travail social, Université du Québec à Montréal,

chercheuse associée, Le Phare, Enfants et Familles.

Il est désormais possible, grâce à des progrès importants dans les soins médicaux, de prolonger la vie d'enfants atteints d'une maladie à issue fatale et de leur permettre de demeurer à domicile (Liben et al., 2000; Kirk, 1998). Ces avancées, telles que la technique de ventilation, la transplantation d'organes, la technique d'hyperalimentation et les nouveaux traitements pharmacologiques, ont cependant entraîné dans leur sillage une complexification des soins ainsi qu'un alourdissement, pour les familles de ces enfants, des responsabilités relatives à leur prise en charge (Trottier, 2004). Ainsi, pour la plupart des familles ayant un enfant gravement malade, les tâches, les inquiétudes, les coûts financiers, la fatigue et l'isolement associés au maintien à domicile de cet enfant deviennent très lourds à supporter (Mongeau et al., 2004; Horsburgh et al., 2002; Liben et al., 2000; Davies et al., 1998b; Kirk, 1998). Parmi les mesures de soutien pouvant leur permettre de mieux faire face aux nombreux défis que comporte leur vie quotidienne, l'offre de répit est primordiale (Davies et al., 2004; Mongeau et al., 2004; McCormack et al., 2000; Gravelle, 1997). Le répit constitue d'ailleurs une composante essentielle des soins palliatifs pédiatriques (ACT et al., 2003; Horsburgh et al., 2002; American Academy of Pediatrics, 2000; Davies et al., 1998b). Il est possible pour des bénévoles de répondre à une partie des besoins de répit de ces familles lorsqu'ils sont soigneusement sélectionnés, formés et supervisés (ACSP et al., 2004; ACT et al., 2003; Horsburgh et al., 2002; Davies et al., 1998a).

En janvier 2001, Le Phare, Enfants et Familles, un organisme sans but lucratif situé à Montréal et voué au soutien de parents d'enfants gravement malades, a mis sur pied le programme "Répit à domicile» offert gratuitement par des bénévoles. Les objectifs en sont principalement de procurer des moments de ressourcement aux familles et des activités de loisirs aux enfants malades (Mongeau et al., 2001). Chaque famille est jumelée à un bénévole qui s'engage à rendre visite à l'enfant à chaque semaine pendant une période d'au 
moins six mois. Dès les débuts de «Répit à domicile», Le Phare a proposé à ses aspirants bénévoles un programme de formation assez satisfaisant dans l'ensemble, mais comportant toutefois de nombreux aspects susceptibles d'être améliorés, cette situation s'expliquant notamment par la rareté des ressources pour soutenir la formation de bénévoles dans un contexte de soins palliatifs pédiatriques. En effet, très peu de ressources sont disponibles à ce sujet malgré la spécificité de ces soins et les recommandations émanant de divers organismes quant au caractère primordial de la formation des bénévoles dans ce domaine (ACSP et al., 2004; ACT et al., 2003). En outre, contrairement à la formation des bénévoles accompagnant des adultes en soins palliatifs, la formation des bénévoles en soins palliatifs pédiatriques est un sujet qui n'a pas été étudié et qui a été très peu commenté ${ }^{2}$. Ces facteurs ont conduit Le Phare à accepter, en 2003, l'offre de l'auteure d'entreprendre avec certains de ses membres un processus de rechercheaction relatif à la formation des bénévoles, et ce, dans le contexte de sa recherche doctorale. Cette recherche visait, dans le cadre d'une démarche collaborative de planification de programme de formation, 1) à mieux connaître les besoins de formation de ces bénévoles et 2) à cerner les principales composantes d'un programme de formation initiale leur étant destiné. Cet article présente une partie des résultats relatifs au premier objectif principal de cette recherche en offrant une synthèse des besoins d'apprentissage des bénévoles de «Répit à domicile». Cette recherche apporte une contribution pertinente au domaine des soins palliatifs pédiatriques compte tenu que des organismes et chercheurs ont reconnu, ces dernières années, le besoin d'approfondir la recherche relative à l'éducation des professionnels et des bénévoles dans ce domaine (Bouvette et al., 2004 ; ACT et al., 2003 ; Davies et al., 2003), tout comme la recherche sur le rôle des bénévoles œuvrant auprès d'enfants nécessitant des soins palliatifs (ACSP et al., 2004).

\section{QUELQUES DÉFINITIONS AUTOUR DES NOTIONS DE BÉNÉVOLE ET DE FORMATION}

Selon le Centre d'études et d'information sur le volontariat (1974, cité par Halba et al., 1997), cinq conditions sont nécessaires pour caractériser un bénévole. Ainsi,

le bénévole (ou volontaire) est celui qui s'engage (notion d'engagement), de son plein gré (notion de liberté), de manière désintéressée (notion d'acte sans but lucratif), dans une action organisée (notion d'appartenance à un groupe, à une structure), au service de la communauté (notion d'intérêt commun) (p. 9).

Cette définition a le mérite de mettre en évidence les conditions du bénévolat proposées par de nombreux auteurs (Lamoureux, 2001 ; Brudney, 1999; Bovay et al., 1998; Simonet, 1998; Godbout, 1995), mais ne fait pas état de la dimension relationnelle de l'engagement bénévole. En outre, le recours à l'expression «de manière désintéressée» plutôt que "sans aucune rémunération » peut laisser croire qu'aucune forme de réciprocité n'est possible dans un contexte de bénévolat, ce qui ne correspond pas à la pensée de certains auteurs (Brudney, 1999; Godbout, 1995) ni aux résultats d'une étude portant sur les conditions de l'établissement d'une bonne relation entre le bénévole, l'enfant gravement malade et sa famille (Champagne et al., 2004). Cette étude, effectuée à partir d'entretiens individuels et de groupes de discussion réalisés avec des bénévoles du Phare, met en lumière l'importance de la réciprocité pour ces derniers. Ainsi, la définition du terme bénévole retenue aux fins de cette recherche s'inspire de celle qui est offerte par le Centre d'études et d'information sur le volontariat (1974, cité par Halba et al., 1997), tout en lui ajoutant la dimension de la reconnaissance proposée par Gagnon et al. en 2000 et reprise par Gagnon et al. en 2002. Elle se lit comme suit: "Personne qui s'engage de son plein gré, sans être rémunérée, dans une action organisée, au service de la communauté, cette action lui permettant de vivre un moment privilégié de reconnaissance de soi et de l'autre.»

La formation est souvent définie comme l'«ensemble des activités, des situations pédagogiques et des moyens didactiques ayant comme objectif de favoriser l'acquisition ou le développement de savoirs (connaissances, habiletés, attitudes) en vue de l'exercice [d'un rôle]» (Legendre, 1993, p. 622). Toutefois, certains auteurs jugent cette définition réductrice. Ainsi, Ferry (1987) explique:

Il me semble important [...] de distinguer nettement la formation des actions éducatives qui en sont les moyens (parmi d'autres) et avec lesquels elle est couramment confondue. La formation ne saurait être réduite à une action exercée par un formateur sur un "formé » malléable, recevant passivement la forme que lui imprime le formateur (p. 37).

Cette distinction vise en fait à ramener la formation à son sens premier qui est, toujours selon cet auteur, celui d'un processus de développement personnel. Elle vise également à ramener le rôle de formateur à celui de médiateur, éloignant ainsi le formateur d'un fantasme de toutepuissance qu'il pourrait manifester à l'endroit du «formé». D’après Ferry (1987), il est plus approprié de définir la formation comme un «processus de développement individuel tendant à acquérir ou à perfectionner des capacités»(p. 36). Dans un autre texte, Ferry (1994, cité par Martin et al., 1996) précise que ce processus s'inscrit dans une finalité pouvant consister, par exemple, en l'obtention d'une qualification ou la possibilité de tenir un rôle social ou professionnel. D'autre part, plusieurs auteurs jugent que la formation des bénévoles doit comprendre différents volets dont l'orientation, la formation initiale, la formation complémentaire, la formation sur mesure et la formation continue (Le Lann, 2003; Spencer-Gray, 2002; Brudney, 1999; Cummings, 1998; Rothstein et al., 1997 ; Brenner, 1993). Dans le contexte de cette recherche, c'est la formation initiale qui était à l'étude et la définition qui en a été retenue s'inspire de celle offerte par Brudney (1999) et de la définition du terme formation proposée par Ferry (1987). Elle se lit comme suit: "Préparation spécifique au rôle que souhaite jouer le bénévole, incluant l'acquisition ou le perfectionnement des habiletés, des connaissances et des attitudes requises pour l'exercice de ce rôle. » Ainsi, l'emploi de l'expression formation initiale ne signifie pas que le futur bénévole ne possède au départ aucune des habiletés, connaissances ou attitudes nécessaires à l'exercice de son rôle. Au contraire, il importe de reconnaître que chaque bénévole possède un bagage d'expériences et d'acquis avant même de s'engager dans la formation.

Par ailleurs, Ferry (1994, cité par Martin et al., 1996) souligne la nécessité des dispositifs de formation pour soutenir le processus de formation, pour lui permettre de se déployer, puisque «ce processus n'est possible que grâce à des médiations » (p. 24). L'expression «programme de formation » a été utilisée dans cette recherche lorsque référence était faite à l'ensemble des composantes visant à soutenir le processus d'acquisition ou de perfectionnement des capacités des futurs bénévoles. Parfois, l'utilisation de l'expression «session de formation» a été préférée, une session de formation étant ici entendue comme un ensemble de huit séances de formation complémentaires d'une durée de trois heures chacune.

En dernier lieu, il convient de définir la catégorie de besoins de formation qui est traitée dans cet article, c.-à-d. les besoins d'apprentissage ${ }^{3}$. Selon Lapointe (1992), ces derniers «s'expriment en termes d'objectifs d'apprentissage (connaissances, 
L'EMPLOI DE L'EXPRESSION FORMATION INITIALE

NE SIGNIFIE PAS QUE LE FUTUR BÉNÉVOLE NE POSSÈDE

AU DÉPART AUCUNE DES HABILETÉS, CONNAISSANCES

OU ATTITUDES NÉCESSAIRES À L'EXERCICE DE SON RÔLE.

AU CONTRAIRE, IL IMPORTE DE RECONNAÎTRE

QUE CHAQUE BÉNÉVOLE POSSĖDE UN BAGAGE

D'EXPÉRIENCES ET D'ACQUIS AVANT MÊME DE S'ENGAGER

DANS LA FORMATION.

attitudes, habiletés) à inclure dans un éventuel programme» (p. 54). Ces objectifs peuvent être définis de manière plus ou moins générale, selon qu'il soit question d'établir les finalités éducatives d'un programme, ses buts ou ses objectifs spécifiques.

\section{CADRE MÉTHODOLOGIQUE}

Une forme de recherche-action pratique associée au courant interprétatif, soit la recherche-action communautaire (community-based action-research) proposée par Stringer (1999), a inspiré le déroulement de cette recherche.

La recherche-action communautaire est une approche collaborative de recherche ou d'investigation qui offre aux gens confrontés à des problèmes spécifiques les moyens de prendre des actions systématiques pour les résoudre. Cette approche de recherche favorise les procédures consensuelles et participatives qui rendent les gens capables (a) d'examiner leurs problèmes de manière systématique,

(b) de formuler des comptes rendus approfondis et détaillés de leurs situations et (c) de concevoir des plans pour faire face aux problèmes soulevés (p. 17, traduction libre).

C'est une adaptation de la notion de chercheur collectif telle qu'elle est proposée par Barbier (1996) qui a été retenue afin d'inspirer le fonctionnement du comité responsable de la recherche 4 . Le chercheur collectif comprenait trois bénévoles, trois employées du Phare et une formatrice, tous associés au programme "Répit à domicile», ainsi qu'une chercheuse responsable du projet, l'auteure de cet article. Sur une période de deux ans, les membres du chercheur collectif se sont rencontrés à seize reprises et ont communiqué très souvent par courrier électronique et par téléphone. L'ensemble des membres a contribué à toutes les étapes de la recherche, à l'exception de l'élaboration de la problématique et du cadre conceptuel, et de la rédaction du rapport qui ont été effectués par la chercheuse responsable du projet. Les autres membres ont toutefois été informés, dès la première réunion, du contenu de la problématique et du cadre conceptuel. Ils ont aussi pris connaissance des objectifs de la recherche et les ont approuvés. De manière plus précise, le chercheur collectif a participé à l'élaboration du devis méthodologique, à la création des instruments de recherche ainsi qu'à la collecte, l'analyse et l'interprétation des données. Finalement, tous les membres ont contribué, mais de manières variées, à l'action au cœur de cette recherche, soit la planification d'une version améliorée du programme de formation des bénévoles de "Répit à domicile».

Le processus de recherche proposé par Stringer (1999), nommé « routine de base de la recherche-action communautaire» (basic community-based action research routine, p. 18), est constitué des étapes d'observation, de réflexion et d'action (the «look, think, act» routine, p. 19). Dans le contexte de cette recherche, la démarche préconisée par Stringer (1999) a été opérationnalisée par la chercheuse responsable du projet sous la forme de scénarios méthodologiques suggérant différentes méthodes pouvant faciliter l'atteinte des objectifs spécifiques de la recherche, pour ensuite être proposée aux membres du chercheur collectif. Cette proposition est demeurée ouverte tout au long de la recherche et soumise à de nombreux changements et ajustements, ainsi qu'il est nécessaire de le faire en recherche-action. Voici, pour chacun des objectifs spécifiques de la recherche, les méthodes et techniques qui ont finalement été retenues par le chercheur collectif.

Le premier cycle de la recherche visait l'atteinte des deux premiers objectifs spécifiques: analyser les besoins de formation des bénévoles du programme Répit à domicile et déterminer les éléments à retenir dans la composition d'un programme de formation initiale leur étant destiné. Pour arriver à déterminer les besoins de formation des bénévoles sous forme d'objectifs d'apprentissage (besoins d'apprentissage) et de méthodes et ressources de formation à privilégier (besoins d'intervention), les principales techniques utilisées ont été l'entretien téléphonique avec cinq parents bénéficiant de répit à domicile, le groupe nominal avec huit bénévoles expérimentés et une employée, ainsi que l'entretien individuel semi-structuré avec sept formatrices. Par la suite, le chercheur collectif a tenu quelques rencontres afin de retenir, parmi toutes les idées émises, les objectifs, méthodes et ressources de formation constituant la base du programme. C'est à partir de cette base que les formatrices ont chacune été invitées à élaborer un plan de formation, entreprenant ainsi l'étape d'action du premier cycle de cette recherche. À partir des huit plans de formation ont été produits huit fascicules destinés aux bénévoles. En complément ont été conçus un guide de ressources pour les bénévoles ainsi que six vidéos. Le deuxième cycle de la recherche correspondait pour sa part à l'objectif spécifique suivant: évaluer comment se déroulait l'implantation du programme de formation auprès d'une cohorte de bénévoles. Les méthodes choisies lors de ce cycle ont été l'observation des huit séances de formation offertes en novembre 2005, le questionnaire d'évaluation de chaque séance de formation et de l'ensemble de la session de formation (remplis par un nombre de 10 à 15 participantes selon les séances), et les entretiens individuels avec sept formatrices. Quant au troisième et dernier cycle de la recherche, il était associé à l'objectif spécifique suivant: examiner, une fois que les bénévoles auraient été impliqués auprès de familles, dans quelle mesure les composantes du programme de formation étaient adaptées à l'exercice de leur rôle. La méthode alors retenue a consisté en un groupe de discussion ayant pris place quatre mois après la session d'implantation de la nouvelle version du programme, avec cinq participantes ayant entre-temps été jumelées à des familles. À la suite de ces deux types d'évaluation, le programme a été amélioré en tenant compte des résultats recueillis, ce qui, tant pour le deuxième que le troisième cycle de recherche était associé à l'étape d'action. Finalement, signalons qu'une démarche continue d'analyse du processus de recherche et d'action a permis l'atteinte du cinquième et dernier objectif spécifique qui consistait à analyser le processus collaboratif vécu par les participants à cette recherche ${ }^{5}$. Toutes les données qualitatives recueillies aux diverses étapes de cette recherche ont été analysées à partir 
d'une stratégie de questionnement analytique ou par analyse thématique (Paillé et Mucchielli, 2003).

Le recours à différents types de triangulation ainsi que l'adoption de divers moyens pouvant favoriser l'atteinte des critères de rigueur de la recherche-action (Savoie-Zajc, 2001) se sont portés garants de la rigueur scientifique de cette recherche. L'usage de la triangulation s'est fait de plusieurs manières: l'emploi de plusieurs méthodes de collecte des données, auprès de diverses catégories de participants et sur une durée d'environ un an et demi, a offert la possibilité de recourir à la triangulation méthodologique, à la triangulation des données et à la triangulation temporelle, tandis que l'interprétation des résultats par les membres du chercheur collectif a permis qu'il y ait triangulation du chercheur (Savoie-Zajc, 1996). Pour ce qui concerne l'atteinte du critère de respect des valeurs et des principes démocratiques et du critère de faisabilité (Savoie-Zajc, 2001), les rencontres régulières du chercheur collectif et les communications écrites fréquentes entre ses membres ont encouragé l'inclusion des participants et ont permis d'objectiver la démarche de recherche afin que des réajustements soient faits au besoin. Quant au critère de cohérence systémique (Savoie-Zajc, 2001), son atteinte a été favorisée par une description détaillée des méthodes et techniques de collecte et d'analyse des données utilisées ainsi que le recours à la triangulation. Enfin, la tenue d'un journal de bord par la responsable de la recherche, la rédaction des comptes rendus des réunions du chercheur collectif et une description détaillée du contexte ont considérablement enrichi la documentation de la démarche et ont ainsi facilité l'atteinte du critère de fiabilité et d'appropriation qui vise à l'explicitation de toute la démarche vécue, afin que les résultats puissent éventuellement être transférables à d'autres milieux et que les participants soient encouragés à poursuivre la démarche (Savoie-Zajc, 2001). Signalons finalement que, sur le plan de l'éthique, cette recherche s'est déroulée conformément aux exigences du programme de doctorat dans lequel elle a été réalisée et que les mesures requises ont été prises afin d'assurer le consentement éclairé des participants, tout comme leur anonymat et la confidentialité des informations recueillies.

\section{RÉSULTATS}

Le tableau 1 propose une synthèse des besoins d'apprentissage des bénévoles de «Répit à domicile» en rapportant les grands thèmes dégagés à partir des idées émises par des parents, des bénévoles et des formatrices. Les idées émises par ces trois groupes de participants sont ici présentées, en même temps que sont signalées les idées ayant été retenues dans l'élaboration du programme de formation des bénévoles de « Répit à domicile».

\section{LES IDÉES DES PARENTS}

\section{RELATIVEMENT AUX BESOINS}

\section{D'APPRENTISSAGE DES BÉNÉVOLES}

Les cinq mères interrogées ont toutes souligné, dans un premier temps, que la formation des bénévoles du programme «Répit à domicile» constitue pour elles une nécessité et se trouve à la base du sentiment de confiance qu'elles ont développé envers leurs bénévoles respectifs. Pour ce qui concerne les besoins d'apprentissage des bénévoles ou, selon ce qu'il a été demandé aux parents, ce que les bénévoles devraient savoir et savoir faire lorsqu'ils terminent leur participation à la formation, une analyse thématique a permis, dans un premier temps, de dégager trente-quatre idées émises par les parents. En deuxième analyse, onze thèmes principaux ont émergé d'une classification de ces idées. Les thèmes sont présentés au tableau 1, organisé selon les neuf rubriques suivantes: notions de base et rôle du bénévole, communication, aspects psychosociaux, aspects physiques, aspects relatifs au deuil, aspects relatifs au stress, aspects éthiques, aspects éducatifs et, finalement, aspects réflexifs.

D'abord, en lien avec le rôle du bénévole, il est important pour les parents que les bénévoles apprennent à connaître les différentes facettes de leur rôle et à les intégrer à leur action bénévole (thème 1.2 dans le tableau 1). Ainsi, les parents soulignent plusieurs attitudes que les bénévoles doivent apprendre à manifester, telles que la souplesse, le respect, le plaisir de jouer avec les enfants, la flexibilité quant à la disponibilité et la discrétion, cette dernière étant particulièrement essentielle au moment de leur intégration dans les familles. Le respect des attentes du Phare, telles que la fiabilité et la ponctualité, est aussi un point que les parents soulignent. Toujours en lien avec la rubrique "Notions de base et rôle du bénévole», les parents soulèvent également le thème de la prise de conscience, par les bénévoles, de la portée de leur action (1.3). Une mère précise: «C'est important qu'ils sachent qu'ils sont appréciés comme ils ne peuvent même pas l'imaginer. »

Tous les parents ont soulevé, d'une manière ou d'une autre, des éléments se rapportant à l'importance pour les bénévoles de savoir communiquer et, surtout, de savoir écouter. Ce besoin des parents d'être écoutés, sans jugement et avec compassion, transparaît avec force dans ce témoignage d'une mère.
La grande qualité que je retrouve chez les bénévoles, c'est leur sens de l'écoute. Nous autres, on trouve ça formidable quelqu'un qui puisse donner de son temps. C'est incroyable, c'est tellement apprécié, parce que c'est quelque chose de rare, c'est tellement rare... Parce que souvent dans la famille, c'est pas tout le monde qui a le temps, ou qui a l'écoute, puis qui a la facilité avec la maladie, qui sont capables. [...] Les parents, ce qui est difficile, c'est qu'on a des up puis des down, parce qu'on a des périodes d'anxiété, puis... C'est pas tout le monde qui sont capables, loin de là, c'est ça l'affaire.

Ainsi, pour les parents, les bénévoles devraient pouvoir mettre en pratique des habiletés d'écoute (2.1), être sensibilisés à l'importance de la communication non verbale (2.2) et, aussi, éviter les comportements nuisant à la communication (2.3). Concernant ce dernier thème, une mère explique que les bénévoles ne doivent pas «donner de faux espoirs aux parents. $\mathrm{Ne}$ pas dire: "Ah! ça va aller mieux, ils vont trouver quelque chose [un traitement]". Ça là, ça use, ça use. Je sais bien que ça part d'une bonne intention, mais ça pour les parents, c'est dur à gérer ».

Pour deux des parents interrogés, il importe également que les bénévoles soient sensibilisés au vécu des enfants et de leurs proches, que cela soit par rapport aux différentes problématiques liées à la maladie (4.1) ou encore par rapport à la réalité et aux besoins des familles (3.1). Selon trois parents sur cinq, les bénévoles devraient être formés pour offrir des soins physiques de base (4.5) (changement de couche, bain, alimentation - quand l'enfant s'alimente normalement). Néanmoins, selon les deux autres parents interrogés, les bénévoles n'ont pas à offrir de soins physiques. Comme l'explique une de ces mères, les bénévoles ne vont pas visiter l'enfant dans le but de lui donner des soins, mais dans le but de lui faire vivre des moments agréables et ce serait d'ailleurs trop leur demander que d'exiger d'eux qu'ils offrent des soins, même de base :

Si elle [la bénévole] a le goût de se promener avec [l'enfant] puis de le tenir, elle va le faire, mais je n'ai pas à lui demander de le repositionner dans sa chaise. Peut-être que ça, ça fait partie de la vie de l'enfant, ça fait que peut-être qu'il faudrait qu'elle l'apprenne, mais donner le bain, puis faire des... Mais, non. Elle est là pour le plaisir de l'enfant je pense, à moins que ce soit son jeu préféré, mais elle n'a pas à donner un repas ou à le nourrir. [...] Déjà qu'elle est là... 
Un seul parent émet le souhait que les bénévoles puissent recevoir de la formation quant à l'offre de soins complexes et qu'ils apprennent, par exemple, à offrir un gavage et à donner des médicaments. Tout en précisant que ce n'est pas le genre de soins dont a besoin son enfant, cette mère explique son point de vue sur les soins physiques qui pourraient être offerts par les bénévoles:

Quelqu'un [...] qui est pris chez eux, qui n'a pas d'aide, [...] je veux dire, ça serait le fun qu'il y ait un bénévole ou deux, qu'il y en ait quelques-uns qui sachent comment faire un gavage, des choses comme ça qui pourraient aider ces personnes-là au moins de temps en temps, pour que ces personnes-là puissent sortir de chez eux.

Elle précise par la suite que cet aspect de la formation pourrait être complémentaire, c'est-à-dire qu'il ne s'adresserait qu'aux bénévoles qui se sentiraient à l'aise d'offrir ce type de soins et que, lorsque ce serait le cas, il faudrait que les parents acceptent de dégager le bénévole de toute responsabilité. Toutefois, puisque les règles du Phare interdisent aux bénévoles d'offrir des soins physiques à cause des risques qui sont liés à une telle offre et parce que Le Phare juge que ces tâches doivent relever de personnel rémunéré, les trois idées regroupées sous le thème "offrir des soins physiques » n'ont pu être retenues.

Aucun parent n'a évoqué le fait qu'il serait important pour les bénévoles d'être familiarisés avec des notions concernant la mort et le deuil, la gestion du stress lié à l'exercice de leur rôle ou, encore, les aspects éthiques de leur rôle, tels que la confidentialité.

C'est la rubrique "Aspects éducatifs» qui remporte la palme quant au plus grand nombre d'idées émises par les mères interrogées. Ainsi, les thèmes organiser et animer des activités récréatives (8.1) et manifester des attitudes éducatives envers les enfants (8.2) apparaissent particulièrement importants aux yeux des parents. Pour les cinq mères interrogées, les bénévoles doivent connaître de nombreuses idées d'activités variées et adaptées aux capacités des enfants, et être capables de jouer avec eux tout en manifestant à leur égard des attitudes de respect, de souplesse et de valorisation. En outre, une mère spécifie que les bénévoles ne doivent "pas les traiter [les enfants] comme des enfants différents». Ils doivent les traiter «à leur rythme, avec leurs capacités, mais comme des enfants normaux. Tu sais, pas les gâter, leur passer tous leurs caprices. [...] On essaye de les éduquer le plus possible dans la normalité ». Un dernier thème relatif à cette rubrique est le recours, par les bénévoles, à leur créativité (8.4). En effet, selon une des mères, les bénévoles doivent apprendre à être inventifs et imaginatifs.

Finalement, deux mères mentionnent qu'il importe que les bénévoles se sentent rassurés face à leurs craintes de rencontrer des enfants malades. Puisque cela implique, selon nous, que les bénévoles puissent dans un premier temps examiner quelles sont leurs craintes, cette idée a été rattachée au thème approfondir sa connaissance de soi (9.1) et classée sous la rubrique des aspects réflexifs.

\section{LES IDÉES DES BÉNÉVOLES \\ RELATIVEMENT À LEURS}

BESOINS D'APPRENTISSAGE

Lors du groupe nominal tenu en septembre 2004, les huit bénévoles et l'employée présents ont proposé soixante-quatre idées d'objectifs d'apprentissage. Une analyse thématique a permis de faire des regroupements parmi ces idées et d'en dégager vingt thèmes principaux, tous présentés au tableau 1 . Les participants ont repris à leur compte tous les thèmes soulevés par les parents sauf celui concernant l'importance de la communication non verbale et le thème concernant l'offre de soins physiques.

Plusieurs des thèmes déjà déterminés à partir des idées des parents ont été enrichis grâce aux idées émises lors du groupe nominal. Par exemple, lorsqu'il est question de la connaissance par les bénévoles des différentes facettes de leur rôle (1.2), de nombreuses idées d'objectifs d'apprentissage ont été fournies par rapport à l'intégration des bénévoles dans les familles. Ainsi, cette étape apparaît cruciale pour les bénévoles qui mentionnent que leur participation à la formation devrait les amener à: «savoir comment intégrer une famille; connaître les types de réactions que peuvent avoir les membres de la famille face à l'arrivée du bénévole dans la famille; savoir comment approcher l'enfant et les parents », et, finalement, « connaître les attentes de la famille». Également en rapport avec la connaissance par les bénévoles des facettes de leur rôle, le groupe nominal a permis d'enrichir la rubrique des aspects éthiques qui était demeurée vide après l'analyse des idées des parents. Ainsi, il a été soulevé que les bénévoles devaient, en fin de formation, «connaître comment le code de déontologie circonscrit leur rôle» et être en mesure de "respecter la confidentialité». Toujours en lien avec l'enrichissement de thèmes qui avaient déjà été déterminés à partir des idées des parents, c'est par rapport au thème approfondir sa connaissance de soi (9.1), appar- tenant aux aspects réflexifs, que l'apport du groupe nominal a été le plus déterminant. Ainsi, pas moins de six idées ont été ajoutées à ce thème, en relation avec les motivations et intérêts du bénévole envers ce type de bénévolat, la reconnaissance de ses propres limites, l'importance de garder ses couleurs et son authenticité, et la connaissance de son propre rapport à la maladie, à la mort et au deuil.

Parmi les onze nouveaux thèmes qui émergent des idées du groupe nominal, le premier est connaître l'organisme et en utiliser les ressources (1.1). Deux des nouveaux thèmes, qui concernent la communication, ont la particularité de n'avoir été soulevés que par les bénévoles. Il s'agit de savoir décoder la dynamique familiale et s'y adapter (2.4) et de mettre en pratique des habiletés de gestion de crise (2.5). Un quatrième thème est relatif à la sensibilisation des bénévoles à la diversité culturelle des familles et il a été placé à la fois sous la rubrique des aspects psychosociaux (3.2) et sous celle des aspects relatifs au deuil (5.3). Par rapport aux aspects physiques, les bénévoles ont mentionné quelques idées associées aux deux thèmes suivants: connaître des notions de base sur les maladies des enfants et leurs effets (4.2) et se sentir plus familiers avec l'équipement médical (4.3). Trois des nouveaux thèmes ont été placés sous la rubrique des aspects relatifs au deuil qui, rappelons-le, était demeurée vide à la suite de l'analyse des idées proposées par les parents. Parmi les idées émises à ce sujet lors du groupe nominal, certaines ont trait à la considération, par le bénévole, des différents deuils susceptibles de se produire dans le contexte de sa relation avec un enfant et sa famille (5.2). Les deux autres thèmes sont la connaissance de notions de base sur le deuil (5.1) et, comme cela a été mentionné précédemment, la sensibilisation à la diversité culturelle des familles (5.3). Plusieurs des idées des bénévoles étaient relatives aux aspects éducatifs. Deux nouveaux thèmes sont proposés sous cette rubrique, soit la gestion du temps de la visite (8.3) et la connaissance de notions de base sur le développement de l'enfant (8.5). Finalement, le onzième thème, qui est en lien avec la capacité pour les bénévoles de faire des retours sur leur action bénévole (9.2), est propre à ces derniers puisqu'il n'a pas émergé des idées des parents et des formatrices.

\section{LES IDÉES DES FORMATRICES \\ RELATIVEMENT AUX BESOINS \\ D'APPRENTISSAGE DES BÉNÉVOLES}

Les quatre-vingt-cinq objectifs proposés par les formatrices lors de la première série d'entretiens auxquels elles ont participé ont pu être regroupés sous vingt 
et un thèmes, parmi lesquels dix-huit avaient déjà été soulevés par les parents et les bénévoles. Certains de ces thèmes ont toutefois été enrichis de nouvelles idées. Par exemple, en lien avec le thème connaître les différentes facettes de son rôle de bénévole et les intégrer à son action bénévole (1.2), l'objectif «Définir le répit» a été proposé par une des formatrices. De la même manière, l'objectif «Définir les soins palliatifs pédiatriques » a été ajouté sous connaître des notions de base sur les maladies des enfants et leurs effets (4.2).

Pour ce qui est des thèmes ayant été soulevés par les parents et les bénévoles, mais non par les formatrices, il y a celui de l'offre de soins physiques qui a émergé des idées des parents mais qui, de la même manière que pour les bénévoles et pour la même raison, n'a pas été mentionné par les formatrices. Et puis, celles-ci n'ont pas soulevé trois des thèmes déterminés à partir des idées des bénévoles, c'est-à-dire savoir décoder la dynamique familiale et s'y adapter, mettre en pratique des habiletés de gestion de crise et faire des retours sur son action bénévole.

Relativement aux trois nouveaux thèmes ayant émergé des idées des formatrices, le premier se trouve sous la rubrique de la communication et a trait à la mise en pratique d'habiletés de relation d'aide (2.6). Un thème a été ajouté à la rubrique des aspects physiques, soit adopter un comportement sécuritaire face aux enfants nécessitant des soins complexes (4.4), qui englobe des idées relatives aux précautions à prendre lors d'interactions avec des enfants qui ont besoin de soins complexes. Finalement, le dernier thème spécifique aux formatrices concerne le développement, par le bénévole, de moyens d'adaptation aux facteurs de stress propres à son action bénévole (6.1).

\section{IDÉES RETENUES POUR LE}

\section{PROGRAMME DE FORMATION ${ }^{6}$}

À l'exception des trois idées relatives à l'offre de soins physiques, toutes les idées mentionnées par les parents ont été retenues dans l'élaboration du programme de formation, bien qu'elles l'aient été à des niveaux de généralité différents. Ainsi, plusieurs idées ont servi à préciser des éléments de contenu plutôt que des objectifs. Pour ce qui concerne les idées proposées par les bénévoles, elles ont toutes été prises en considération lors de l'élaboration du programme de formation. Cependant, certaines idées ont été traitées de manière moins approfondie que d'autres. Par exemple, pour ce qui concerne le thème de la gestion de crise, il n'a pas semblé approprié de transmettre aux bénévoles des notions sur la gestion de crise, mais des mises en situation illustrant divers types de crises pouvant être vécues par les familles ont été proposées. En ce qui a trait aux idées des formatrices, la grande majorité ont été reprises. Toutefois, les quatre objectifs regroupés sous le thème de la mise en pratique d'habiletés de relation d'aide ont été abandonnés lors du choix définitif des objectifs du programme de formation en raison du caractère trop professionnel de la relation d'aide dans le contexte du rôle joué par les bénévoles de "Répit à domicile». Le concept même de relation d'aide a été remplacé par celui d'écoute active, qui a paru beaucoup plus près des idées soumises par les parents et les bénévoles. Finalement, les deux objectifs proposés en lien avec le thème de la gestion du stress ont été retirés, étant donné que les bénévoles n'ont pas soulevé ce besoin et qu'il est apparu que ces objectifs pourraient être proposés à l'intérieur de séances de formation continue.

La pertinence des idées retenues pour la formation été confirmée une première fois quand il a été demandé aux personnes ayant participé à la nouvelle version du programme de formation, à l'automne 2005, d'indiquer dans le questionnaire d'évaluation distribué à la fin de chaque séance ce qu'elles en avaient retenu de plus important. Les participantes ont alors souligné des éléments se rapportant pour une large part à l'organisation et à l'animation des activités récréatives, aux attitudes éducatives à démontrer envers les enfants, à la connaissance des différentes facettes de leur rôle, à l'adoption d'un comportement sécuritaire compte tenu de la condition médicale des enfants, aux habiletés d'écoute, et aux réactions et besoins des enfants et des familles. Par la suite, les cinq bénévoles ayant participé au groupe de discussion ont expliqué que deux apprentissages majeurs se dégageaient parmi ceux qu'elles avaient intégrés à leur pratique bénévole. Le premier était qu'à titre de bénévoles, elles n'ont pas à intervenir dans le fonctionnement des familles, et le deuxième se rapportait à l'importance de considérer les enfants avec lesquels elles sont jumelées d'abord à travers leurs forces, leurs capacités et leurs désirs plutôt qu'à travers la maladie et les incapacités qu'elle entraîne.

\section{DISCUSSION}

Concernant les besoins d'apprentissage des bénévoles de «Répit à domicile », c'est la question de l'offre de soins physiques par les bénévoles qui sera d'abord discutée. Comme cela a été mentionné précédemment, les idées relatives à l'offre de soins physiques sont les seules idées soumises par les parents à ne pas avoir été prises en compte dans l'élaboration du programme de formation, et ce, parce que les règles du
Phare interdisent aux bénévoles de prodiguer des soins physiques en raison des risques associés à ces gestes. Toutefois, les besoins exprimés par certains parents à cet égard mettent en évidence, tout comme cela a déjà été souligné dans plusieurs recherches, que l'offre de répit à ces familles doit être multiple et inclure la possibilité de recourir aux services de professionnels en mesure de prodiguer des soins (Mongeau et al., 2004; Olsen et al., 2001; McCormack et al., 2000). Par ailleurs, le fait que des parents proposent que les bénévoles soient préparés à offrir des soins physiques indique une certaine difficulté de leur part à distinguer les tâches relevant des bénévoles de celles relevant de professionnels. Il faut bien admettre que la limite n'est pas toujours claire pour ce qui est des soins de base (ex. : alimentation, changement de couche, bain), mais ne l'est-elle pas en ce qui a trait aux soins complexes (ex.: offre de médicaments, gavage) ? Pour des parents ayant eux-mêmes appris à donner des soins à leurs enfants, peut-être apparaît-il adéquat que des bénévoles soient appelés à agir de la même manière qu'eux? Cela confirme l'importance pour un organisme comme Le Phare d'instaurer, parallèlement à la formation de ses bénévoles, divers moyens d'information à l'endroit des parents concernant ce qui peut être attendu ou non d'un bénévole. Toujours en lien avec les soins physiques, il peut paraître surprenant de constater que les parents n'ont pas soulevé la nécessité pour les bénévoles de se familiariser avec différents équipements médicaux nécessaires au maintien d'une bonne condition chez les enfants. Cela est fort probablement attribuable au fait qu'un seul enfant, parmi les cinq enfants des parents interrogés, avait besoin du soutien de la technologie médicale sur une base régulière. L'insécurité des bénévoles face à la technologie médicale est toutefois clairement ressortie de leurs propos et les formatrices ont mentionné plusieurs idées se rapportant à la familiarisation des bénévoles avec l'équipement médical ainsi qu'à l'adoption de comportements sécuritaires par ces derniers.

Sur un autre plan, le caractère primordial du développement d'habiletés de communication chez les bénévoles est ressorti avec force des idées proposées par tous, ce qui rejoint les recommandations de différents organismes en soins palliatifs pédiatriques concernant l'importance d'une bonne communication entre les familles, les professionnels et les bénévoles (ACSP et al., 2004; ACT et al., 2003). Rappelons cependant que le concept de relation d'aide et les principes s'y rattachant ont été exclus $\mathrm{du}$ programme de formation au profit $\mathrm{du}$ 
IL EST RESSORTI CLAIREMENT DES IDÉES AVANCÉES PAR LES PARENTS

QUE LE RÔLE DU BÉNÉVOLE DEVAIT S'ARTICULER AUTOUR DE

DEUX AXES MAJEURS, SOIT L'ÉCOUTE ET L'ANIMATION D'ACTIVITÉS,

ET QUE L'INTRODUCTION PAR LES BÉNÉVOLES D'UNE CERTAINE

NORMALITÉ DANS LA VIE DE LEURS ENFANTS REVÊTAIT

UNE GRANDE VALEUR.

concept d'écoute active, étant donné la volonté du Phare d'éviter tout risque de glisser vers une professionnalisation $\mathrm{du}$ rôle des bénévoles et compte tenu des témoignages des parents qui ont insisté sur l'importance pour les bénévoles de savoir les écouter comme un bon ami le ferait et, notamment, sans se livrer à aucune recherche de solutions.

Autre point d'intérêt: seuls les bénévoles et les formatrices ont mentionné des idées de formation en lien avec la considération par les bénévoles des différents deuils susceptibles de se produire dans le contexte de leurs relations avec les familles. Il faut reconnaître ici que, pour les parents interrogés, le cadre d'un entretien téléphonique avec une personne inconnue se prêtait fort peu à l'évocation de l'issue possiblement fatale de la maladie de leurs enfants. Et puis, il est ressorti clairement des idées avancées par les parents que le rôle du bénévole devait s'articuler autour de deux axes majeurs, soit l'écoute et l'animation d'activités, et que l'introduction par les bénévoles d'une certaine normalité dans la vie de leurs enfants revêtait une grande valeur. Cette perception du rôle du bénévole par les parents, également révélée dans d'autres travaux (Mongeau et al., 2004; Mongeau et al., 2001), explique peut-être en partie pourquoi les parents interrogés n'ont pas associé la formation des bénévoles aux nombreux deuils vécus par l'enfant et la famille ainsi qu'à l'éventualité du décès de l'enfant et à ses répercussions sur le système familial.

L'idée de préparer les bénévoles à mieux faire face aux facteurs de stress propres à leur action auprès des enfants et des familles n'a été mentionnée ni par les parents ni par les bénévoles. Face à cela, il convient de mettre en doute l'idée largement répandue selon laquelle le bénévolat en soins palliatifs entraîne nécessairement une augmentation importante du niveau de stress chez celles et ceux qui le pratiquent. Dans les faits, certaines recherches tendent à démontrer que ce n'est pas le cas (Field et al., 1993) ou que les principaux facteurs de stress sont davantage liés au travail en équipe interdiscipli- naire qu'aux contacts avec les personnes malades et leurs proches (Berteotti et al., 1994; Paradis et al., 1987). Ainsi donc, s'il importe que la gestion du stress fasse l'objet de formation pour les professionnels en soins palliatifs - comme le montre une étude récente concernant les besoins de formation d'infirmières du Québec (Fillion et al., 2005) - cela ne semble pas aussi prioritaire pour les bénévoles. Plusieurs différences, dont les suivantes, sont ici à prendre en considération: les bénévoles œuvrant en soins palliatifs le font par choix et non par obligation, ils n'ont ni les mêmes tâches ni autant de responsabilités que les professionnels et ils ne sont pas liés aux mêmes attentes du milieu pour ce qui concerne l'efficacité de leurs actions (Gill, 2002).

Finalement, soulignons que des idées relatives aux aspects réflexifs de la formation des bénévoles ont émergé très majoritairement du discours de ces derniers, très peu du discours des formatrices et pas du tout du discours des parents. Cela illustre le sérieux avec lequel la plupart des bénévoles considèrent leur engagement et aussi le désir, pour plusieurs, d'approfondir leur connaissance d'eux-mêmes.

\section{LA SPÉCIFICITÉ DE LA FORMATION DES BÉNÉVOLES EN SOINS PALLIATIFS PÉDIATRIQUES}

En conclusion à cette discussion, il convient d'examiner en quoi peuvent se distinguer les besoins d'apprentissage de bénévoles œuvrant auprès d'enfants en soins palliatifs par rapport aux besoins de ceux qui œuvrent auprès d'adultes. Les programmes de formation destinés aux bénévoles en soins palliatifs proposent généralement des éléments de contenu relatifs aux grands thèmes suivants: les besoins des personnes malades et de leurs proches, la perte et le deuil, les habiletés de communication, et la connaissance de soi et de ses attitudes face à la mort et au deuil (Champagne, 2007). Nous l'avons vu, des idées relatives à chacun de ces thèmes ont pu être dégagées à partir de l'analyse des besoins d'apprentissage des bénévoles de «Répit à domicile». Cependant, la spécificité des soins palliatifs pédiatriques fait que chacun de ces thèmes, lorsqu'il est abordé avec des bénévoles qui vont œuvrer auprès d'enfants, doit être traité à partir d'objectifs et d'éléments de contenu adaptés ou même nouveaux.

En ce qui concerne les besoins psychosociaux et physiques des enfants atteints de maladies potentiellement fatales, ils comportent plusieurs différences par rapport à ceux d'adultes dans la même situation. Au départ, œuvrer auprès d'enfants et d'adolescents, même en bonne santé, nécessite une connaissance élémentaire de leur développement. Lorsqu'il s'agit d'accompagner des enfants et adolescents gravement malades, il faut aussi développer une certaine compréhension de leurs réactions face à la maladie et des besoins psychosociaux en découlant. Et puis, les maladies qui affectent ces enfants sont non seulement différentes de celles affectant les adultes, mais aussi beaucoup plus diversifiées, et elles entraînent souvent des incapacités intellectuelles et physiques. De plus, le recours à la technologie médicale peut augmenter la durée de vie de ces enfants de manière considérable (Trottier, 2004; Liben et al., 2000; Kirk, 1998). Lorsqu'il est question d'accompagner des adultes en soins palliatifs, les défis de la diversité des conditions médicales, des incapacités intellectuelles et physiques, et du recours à la technologie médicale se présentent avec beaucoup moins d'acuité. Une autre différence majeure est relative à l'importance du système familial dans la vie de l'enfant (Mongeau et al., 2004; Horsburgh et al., 2002; Liben et al., 2000; Davies et al., 1998b; Kirk, 1998). Sans nier l'ampleur des répercussions, sur toute famille, de la maladie d'un de ses membres, il demeure que dans le cas d'un enfant, il est souvent plus difficile pour la famille, sinon impossible, de trouver un sens à cette épreuve. Et comme l'évolution de la maladie de l'enfant peut s'étendre sur plusieurs années, le réseau social entourant la famille est plus à risque de s'effriter. En outre, les frères et sœurs sont largement affectés par les événements.

Par rapport aux thèmes du deuil et de la mort, plusieurs aspects sont différents lorsqu'il s'agit d'accompagner un enfant plutôt qu'un adulte: le bénévole doit notamment développer une compréhension élémentaire de l'évolution du concept de mort chez les enfants et les adolescents. Il lui faut aussi mieux connaître les particularités du deuil chez un parent et chez un membre de la fratrie ainsi que se familiariser avec quelques points de repère pour le soutien des familles endeuillées.

C'est toutefois au regard des habiletés de communication que la formation des bénévoles œuvrant auprès d'enfants gra- 


\section{Notions de base et rôle du bénévole}

1.1. Connaître l'organisme et en utiliser les ressources

1.2. Connaître les différentes facettes de son rôle de bénévole et les intégrer à son action bénévole

1.3. Prendre conscience de la portée de son action bénévole

Parents

Bénévoles

Formatrices

2. Communication

2.1. Mettre en pratique des habiletés d'écoute

2.2. Être sensibilisé à l'importance de la communication non verbale

2.3. Éviter les comportements nuisant à la communication

2.4. Savoir décoder la dynamique familiale et s'y adapter (non retenu)

2.5. Mettre en pratique des habiletés de gestion de crise (non retenu)

2.6. Mettre en pratique des habiletés de relation d'aide (non retenu)

$\begin{array}{lll} & \checkmark & \sqrt{ } \\ \sqrt{ } & \sqrt{ } & \sqrt{ } \\ \sqrt{ } & \sqrt{ } & \sqrt{ }\end{array}$

\section{L'enfant malade et ses proches - Aspects psychosociaux}

3.1. Être sensibilisé au vécu des enfants et de leurs proches

3.2. Être sensibilisé à la diversité culturelle des familles

$\begin{array}{lll}\sqrt{ } & \sqrt{ } & \sqrt{ } \\ \sqrt{ } & & \sqrt{ } \\ \sqrt{ } & \sqrt{ } & \sqrt{ } \\ & \sqrt{ } & \\ & \sqrt{ } & \end{array}$

4. L'enfant malade et ses proches - Aspects physiques

4.1. Être sensibilisé au vécu des enfants et de leurs proches

4.2. Connaître des notions de base sur les maladies des enfants et leurs effets

4.3. Se sentir plus familier avec l'équipement médical

4.4. Adopter un comportement sécuritaire face aux enfants nécessitant des soins complexes

4.5. Offrir des soins physiques (non retenu)

\section{Aspects relatifs au deuil}

5.1. Connaître des notions de base sur le deuil

5.2. Considérer différents deuils susceptibles de se produire dans le contexte de sa relation avec l'enfant et sa famille

5.3. Être sensibilisé à la diversité culturelle des familles

5.4. Connaître les différentes facettes de son rôle de bénévole (auprès de l'enfant en fin de vie et de sa famille) et les intégrer à son action bénévole

\section{Aspects relatifs au stress}

6.1. Développer des moyens d'adaptation aux facteurs de stress propres à son action bénévole (non retenu)

\section{Aspects éthiques}

7.1. Connaître les différentes facettes de son rôle de bénévole (en rapport avec les principes éthiques) et les intégrer à son action bénévole

\section{Aspects éducatifs}

8.1. Organiser et animer des activités récréatives

8.2. Manifester des attitudes éducatives envers l'enfant

8.3. Gérer le temps de la visite

8.4. Avoir recours à sa créativité

8.5. Connaître des notions de base sur le développement de l'enfant

\section{Aspects réflexifs}

9.1. Approfondir sa connaissance de soi

9.2. Faire des retours sur son action bénévole

vement malades doit comporter le plus d'aspects nouveaux. En effet, bien que les habiletés de communication, et principalement l'écoute active, puissent s'appliquer tout autant avec des parents d'enfants gravement malades qu'avec des adultes en fin de vie et leurs proches, elles ne sont pas suffisantes pour entrer en relation avec les enfants. C'est ici que la préparation à organiser et animer différents types d'activités récréatives prend tout son sens pour les bénévoles, car ce sont ces activités qui leur permettent d'entrer en communication avec des enfants d'âges et de conditions variés. C'est également ce qui explique l'importance pour ces bénévoles d'avoir largement recours à leur créativité.

Finalement, pour ce qui touche la connaissance de soi et de ses attitudes face à la mort et au deuil, il importe que le béné- vole œuvrant auprès d'enfants gravement malades élargisse sa réflexion. En effet, étant donné que la maladie de ces enfants peut se prolonger sur une longue période et qu'elle entraîne souvent des handicaps, le bénévole se doit de réfléchir également à ses attitudes, sentiments et croyances face à la maladie en elle-même et face aux personnes ayant des besoins particuliers. 


\section{QUELQUES RETOMBÉES DE CETTE RECHERCHE}

Concernant les retombées de cette recherche-action, soulignons d'abord que les parents et les bénévoles ont apprécié les différentes occasions qui leur ont été offertes de faire entendre leurs préoccupations et leurs suggestions. Les besoins des parents de pouvoir compter sur l'écoute de bénévoles attentifs ont été réaffirmés tout comme ceux des enfants de pouvoir être reconnus dans leurs désirs et capacités. En outre, la nécessité de proposer aux familles une gamme élargie d'options de répit, incluant du répit offert par des professionnels en mesure de dispenser des soins physiques, a été réitérée. D’autres retombées de cette recherche-action concernent l'amélioration des pratiques. Ainsi, les pratiques d'accompagnement des bénévoles ont été mieux définies, en même temps que l'ont été leurs besoins d'apprentissage, et elles s'appuient désormais sur une formation plus complète et rigoureuse. La pratique du travail en collaboration, qui n'était pas nouvelle au Phare, a été vécue de manière plus élargie et plus intense, ce qui a entraîné les souhaits de certains que ce genre d'expérience soit répété. Quant à la pratique de la planification de programmes de formation, elle est maintenant mieux maîtrisée par les formatrices et par les membres du chercheur collectif. Finalement, certaines des retombées sont relatives à la création de matériel de formation. De nouveaux modules de formation ont été conçus à partir de la redéfinition de contenus, chacun de ces modules comprenant un plan de formation, un fascicule pour les bénévoles, une vidéo et un questionnaire d'évaluation. Un guide de ressources pour les bénévoles a également été élaboré. Le Phare prévoit diffuser en français et en anglais les documents écrits conçus dans le contexte de cette recherche. De toute évidence, les besoins d'apprentissage déterminés lors de cette recherche ne conviennent pas à toutes les formes de bénévolat en contexte de soins palliatifs pédiatriques. Toutefois, ils constituent une base solide à partir de laquelle différents organismes pourront spécifier les besoins d'apprentissage de leurs propres bénévoles, et ce, également dans des contextes où les bénévoles œuvrent principalement auprès d'adultes, mais où l'on souhaite pouvoir répondre à l'occasion à des demandes provenant de familles d'enfants gravement malades.

La recherche rapportée ici comporte des limites, parmi lesquelles il apparaît important de signaler le nombre restreint de bénévoles consultés lors des premier et troisième cycles de la recherche. En outre, nous sommes conscients que, pour des organismes intéressés à s'inspirer de notre processus de recherche-action, ce dernier peut paraître particulièrement exigeant. Quant aux organismes désirant s'inspirer du programme de formation, ils auront bien entendu à l'ajuster en fonction de leurs contextes respectifs.

Bien des avenues restent à explorer dans le domaine du bénévolat en soins palliatifs. Alors qu'une première maison de soins palliatifs pédiatriques a récemment ouvert ses portes au Québec ${ }^{7}$ et que, déjà, plusieurs maisons et unités de soins palliatifs accueillent des adultes en fin de vie, il devient particulièrement intéressant de s'interroger au sujet des différences et des similarités entre les bénévoles à domicile et ceux œuvrant dans les maisons ou les unités, et ce, en termes de besoins de formation, d'expérience vécue et de sens attribué à l'engagement bénévole. Sur le plan du répit en contexte de soins palliatifs, il importera, dans de futures recherches, d'examiner les retombées pour les familles du répit offert en maison de soins palliatifs comparativement au répit offert à domicile par des bénévoles. Finalement, il serait spécialement pertinent d'examiner quelles sont les raisons qui amènent certaines familles à refuser l'offre bénévole de « Répit à domicile».

\section{Bibliographie}

AMERICAN ACADEMY OF PEDIATRICS - COMMITTEE ON BIOETHICS and COMMITTEE ON HOSPITAL CARE (2000). "Palliative care for children", Pediatrics, vol. $106, \mathrm{n}^{\circ} 2$, p. 351-357.

ASSOCIATION CANADIENNE DE SOINS PALLIATIFS [ACSP] et RÉSEAU CANADIEN DE SOINS PALLIATIFS POUR LES ENFANTS [RCSPE] (2004). Soins palliatifs pédiatriques, Principes directeurs et normes de pratique - Ébauche, Ottawa, Ontario, ACSP, <www.acsp.net/normes_pediatriques_ enquete/ACSP-normes_pediatriques-ebauche1feb2006.pdf $>$, consulté le 7 février 2006.

ASSOCIATION FOR CHILDREN WITH LIFE-THREATENING OR TERMINAL CONDITIONS AND THEIR FAMILIES [ACT] et ROYAL COLLEGE OF PAEDIATRICS AND CHILD HEALTH [RCPCH] (2003). A Guide to the Development of Children's Palliative Care Services, $2^{\mathrm{e}}$ éd., Bristol, Grande-Bretagne, ACT.

BARBIER, R. (1996). La recherche action, Paris, Anthropos / Economica.

BERTEOTTI, C. et D.R. SEIBOLD (1994). "Coordination and role-definition problems in health-care teams: A hospice case study ", dans L. FREY (dir.), Group Communication in Context: Studies of Natural Groups, Hillsdale, NJ, Lawrence Erlbaum, p. 107-131.
BOUVETTE, M., J. PEREIRA et L. LIBRACH (2004). Education for Formal Caregivers: A Health Canada Initiative, conférence présentée au $\mathrm{XV}^{\mathrm{e}}$ Congrès international sur les soins aux malades en phase terminale, Montréal, Québec.

BOVAY, C. et J.-P. TABIN (1998). Les nouveaux travailleurs: bénévolat, travail et avenir de la solidarité, Genève, Labor et Fides.

BRENNER, P.R. (1993). «The volunteer component », dans A. DAILEY-ARMSTRONG et S. ZARBOCK GOLTZER (dir.), Hospice Care for Children, New York et Oxford, Oxford University Press, p. 198-215.

BRUDNEY, J.L. (1999). "The effective use of volunteers: Best practices for the public sector », Law and Contemporary Problems, vol. $62, \mathrm{n}^{\circ} 4$, p. 219-255.

CHAMPAGNE, M. (2007a). Préparer des bénévoles à offrir du répit à des familles d'enfants gravement malades: planification d'un programme de formation dans le contexte d'une recherche-action, Thèse de doctorat inédite, Université du Québec à Montréal, Montréal.

CHAMPAGNE, M. (2007b). «La pratique de la recherche-action: entre utopie et nécessité», dans H. DORVIL (dir.), Problèmes sociaux, Tome III, Théories et méthodologies de la recherche, Québec, Presses de l'Université du Québec, p. 463-490.

CHAMPAGNE, M., S. MONGEAU, M.-C. LAURENDEAU, S. LIBEN et P. CARIGNAN (2004). "Conditions d'une bonne relation entre le bénévole, l'enfant atteint d'une maladie à issue fatale et sa famille », Les Cahiers de soins palliatifs, vol. $5, \mathrm{n}^{\circ} 2$, p. 5-28.

CUMMINGS, I. (1998). "Training of volunteers ", dans D. DOYLE, G. W. C. HANKS et N. MACDONALD (dir.), Oxford Textbook of Palliative Medicine, 2 éd., Oxford, Oxford University Press, p. 1221-1224.

DAVIES, B. et D. HOWELL (1998a). «Special services for children », dans D. DOYLE, G. W. C. HANKS et N. MACDONALD (dir.), Oxford Textbook of Palliative Medicine, $2^{\mathrm{e}}$ éd., Oxford, Oxford University Press, p. $1077-1084$.

DAVIES, B. et R. STEELE (1998b). «Families in pediatric palliative care ", dans R. PORTENOY et E. BRUERA (dir.), Topics in Palliative Care, vol. 3, New York, Oxford University Press, p. 29-49.

DAVIES, B., R. STEELE, K.I. STAJDUHAR et A. BRUCE (2003). "Research in pediatric palliative care », dans R. K. PORTENOY et E. BRUERA (dir.), Issues in Palliative Care Research, New York, Oxford University Press, p. 355-370.

DAVIES, B., R. STEELE, J.B. COLLINS, K. COOK et S. SMITH (2004). "The impact on families of respite care in a children's hospice program », Journal of Palliative Care, vol. $20, n^{\circ} 4$, p. $277-286$.

FERRY, G. (1987). Le trajet de la formation, Paris, Dunod. 
FIELD, D. et I. JOHNSON (1993). «Satisfaction and change: A survey of volunteers in a hospice organisation», Social Science and Medicine, vol. 36, n ${ }^{\circ}$ 12, p. 1625-1633.

FILLION, L., M. FORTIER et R.L. GOUPIL (2005). «Educational needs of palliative care nurses in Quebec », Journal of Palliative Care, vol. $21, \mathrm{n}^{\mathrm{o}} 1$, p. 12-18.

GAGNON, É. et A. FORTIN (2002). "L'espace et le temps de l'engagement bénévole. Essai de définition", Nouvelles pratiques sociales, vol. $15, \mathrm{n}^{\circ} 2$, p. 66-76.

GAGNON, É. et A. SÉVIGNY (2000). «Permanence et mutations du monde bénévole: Note critique", Recherches sociographiques, vol. $41, n^{\circ} 3$, p. 529-544.

GILL, E. (2002). «Unlocking the iron cage: Human agency and social organization », Studies in Symbolic Interaction, vol. 25, p. 109-128.

GODBOUT, J.T. (1995). «Le don entre étrangers », ch. 4 dans L'esprit du don, $2^{\mathrm{e}}$ éd., Montréal, Québec, Boréal, p. 95-114.

GRAVELLE, A.M. (1997). «Caring for a child with a progressive illness during the complex chronic phase: Parents' experience of facing adversity », Journal of Advanced Nursing, vol. 25 , n ${ }^{\circ} 4$, p. $738-745$.

HALBA, B. et M. LE NET (1997). Bénévolat et volontariat dans la vie économique, sociale et politique, Paris, La Documentation française.

HORsburGH, M., A. TRENHOLME et T. HUCKLE (2002). «Paediatric respite care: A literature review from New Zealand", Palliative Medicine, vol. 16, n 2, p. 99-105.

KIRK, S. (1998). «Families' experiences of caring at home for a technology-dependent child: A review of the literature», Child, Care, Health and Development, vol. 24, $\mathrm{n}^{\circ} 2$, p. 101-114.

LAMOUREUX, H. (2001). «Place du bénévolat dans un contexte d'effritement de l'Étatprovidence », Le gérontophile, vol. $23, \mathrm{n}^{\circ} 1$, p. 27-30.

LAPOINTE, J.J. (1992). La conduite d'une étude de besoins en éducation et en formation: une approche systémique, Québec, Presses de l'Université du Québec.

LEGENDRE, R. (1993). Dictionnaire actuel de l'éducation, $2^{\mathrm{e}}$ éd., Montréal, Guérin; Paris, Eska.

LE LANN, P. (2003). «La formation initiale des bénévoles d'accompagnement: un luxe ou une nécessité ? ", Revue JALMALV, $\mathrm{n}^{\circ} 73$, p. 39-43.

LIBEN, S. et B. MONGODIN (2000). La notion de répit pour les familles d'enfants atteints de maladies à issue fatale: Rapport de recherche, Montréal, Québec, Le Phare, Enfants et Familles.

MARTIN, J.-P. et É. SAVARY (1996). Formateur d'adultes - Se professionnaliser, exercer au quotidien, Lyon, France, Chronique Sociale.
MCCORMACK, B., M. IACONO et J. WHITTINGHAM (2000). Advancing the Agenda for Families of Children with Complex Care Needs in Toronto, Toronto, Ontario, Toronto District Health Council/Le conseil régional de santé de Toronto.

MONGEAU, S., P. CARIGNAN, M. CHAMPAGNE, M.-C. LAURENDEAU et S. LIBEN (2004). Au-delà du répit et des activités... un message de solidarité: Évaluation du programme Répit à domicile, Le Phare, Enfants et Familles, Phase II, Montréal, Québec, Université du Québec à Montréal/Le Phare, Enfants et Familles.

MONGEAU, S., M.-C. LAURENDEAU et P. CARIGNAN (2001). Un soutien qui fait une différence: Évaluation de la phase pilote du programme "Répit à domicile», Montréal, Québec, Le Phare, Enfants et Familles / Université du Québec à Montréal/Régie régionale de la santé et des services sociaux de Montréal-Centre.

OLSEN, R. et P. MASLIN-PROTHERO (2001). "Dilemmas in the provision of own-home respite support for parents of young children with complex health care needs: Evidence from an evaluation », Journal of Advanced Nursing, vol. 34, $\mathrm{n}^{\circ}$ 5, p. 603-610.

PAILlÉ, P. et A. MUCCHIELli (2003). L'analyse qualitative en sciences humaines et sociales, Paris, Armand Colin.

PARADIS, L.F., B. MILLER et V.M. RUNNION (1987). «Volunteer stress and burnout: Issues for administrators ", Hospice Journal, vol. $3, n^{\circ} 2 / 3$, p. 165-183.

ROTHSTEIN, J.M. et M.M. ROTHSTEIN (1997). «Education and Training», section VII dans The Caring Community, A Fieldbook for Hospice Palliative Care Volunteer Services, Burnaby, BC, British Columbia Hospice Palliative Care Association, p. 162-197.

SAVOIE-ZAJC, L. (1996). "Triangulation (technique de validation par) », dans A. MUCCHIELLI (dir.), Dictionnaire des méthodes qualitatives en sciences humaines et sociales, Paris, Armand Colin, p. 261-262.

SAVOIE-ZAJC, L. (2001). "La rechercheaction en éducation: ses cadres épistémologiques, sa pertinence, ses limites », dans M. ANADÓN (dir.), Nouvelles dynamiques de recherche en éducation, Québec, Les Presses de l'Université Laval, p. 15-49.

SIMONET, M. (1998). «Le bénévole et le volunteer: Ce que traduire veut dire...", dans Une seule solution, l'association? Socio-économie du fait associatif - La revue $d u$ MAUSS semestrielle, $\mathrm{n}^{\circ} 11$, Paris, La Découverte, p. 57-64.

SPENCER-GRAY, S.-A. (2002). «The training and education of volunteers", dans D. DOYLE (dir.), Volunteers in Hospice and Palliative Care, A Handbook for Volunteer
Service Managers, Oxford, Oxford University Press, p. 52-79.

STRINGER, E.T. (1999). Action Research, $2^{\mathrm{e}}$ éd., Thousand Oaks, Californie, Sage.

TROTTIER, G.D. (2004). Politique en soins palliatifs de fin de vie, Québec, Direction des communications du ministère de la Santé et des Services sociaux, Gouvernement du Québec.

\section{Notes}

1. L'auteure tient à souligner le travail remarquable des membres du chercheur collectif: Gabriel Baril, Emma Comeau, Valérie de Varennes, Stéphanie Dumont remplacée en cours de projet par Annick Gervais, Karine Landerman, Maryse Latendresse et Carole Tétreault.

Une bourse de doctorat du Fonds québécois de la recherche sur la société et la culture (FQRSC) a permis la réalisation de cette recherche, tout comme le soutien indéfectible des deux codirectrices de l'auteure, mesdames Suzanne Mongeau et Lorraine Savoie-Zajc.

2. Cette affirmation s'appuie sur une recension approfondie des écrits faite à partir de l'interrogation des bases de données suivantes: BDSP [Banque de données en santé publique] (1993-2005); ERIC (1992Janvier 2006); FRANCIS (1991-Novembre 2005); MANITOU (système documentaire de l'UQAM) ; PsycINFO (1993 à Janvier 2006); PubMed (1982-Janvier 2006); Repère (1992 à Décembre 2005); Sociological Abstracts (1986-Janvier 2006). Une recherche a aussi été faite sur Internet, à partir du site du Centre canadien de philanthropie, du moteur de recherche Google et du métamoteur de recherche Copernic.

3. Dans la recherche, deux catégories de besoins de formation ont été étudiés, soit les besoins d'apprentissage et les besoins d'intervention. Toutefois, seuls les besoins d'apprentissage sont présentés dans cet article.

4. Il importe de préciser que Barbier décrit le fonctionnement du chercheur collectif à l'intérieur d'une approche spécifique de recherche, soit la recherche-action existentielle, qui présente des distinctions importantes par rapport à l'approche dont nous nous sommes inspirée.

5. Pour plus d'information au sujet de cette démarche, voir Champagne, 2007b.

6. Une liste complète des 87 objectifs du programme de formation des bénévoles de « Répit à domicile» se trouve dans Champagne (2007a).

7. Il s'agit de la Maison André-Gratton, située à Montréal, et relevant du Phare, Enfants et Familles. 